\title{
Error modeling and Sensitivity Analysis of 5-DOF Parallel Tripod Head Dianfan Zhang ${ }^{1}$, Jiang $\mathrm{Hu}^{1}, \mathrm{Yu} \mathrm{Rong}^{2}, \mathrm{Xiaoman}^{1}$, Shuhong Cheng ${ }^{1}$ \\ ${ }^{1}$ School of Electric Engineering, Yanshan University, Qinhuangdao 066004, China, PRC \\ ${ }^{2}$ Hebei Normal University Science \& Technology, Qinhuangdao 066004, China, PRC
}

Keywords: parallel mechanism, kinematics, error modeling, sensitivity analysis.

\begin{abstract}
Aiming at 5-dof parallel tripod head, the integrated parameter modeling of parallel mechanism with less degree of freedom is researched in this paper. Firstly, the kinematics parameters analysis of each component of 5-dof parallel mechanism is carried out and kinematics model of 5-dof mechanism is established, mathematical modeling of 5-dof mechanism error then is systematically proposed with integrated modeling method within the identical framework, which constructs the corresponding relationship between end-effector's pose errors and geometric error and clearly discloses the influence of geometric error source on end effector's uncontrollable errors. Finally, by the given the structure parameters, distribution rule of the uncontrollable errors coming from different height of workspace can be figured out with Monte Carlo simulation method, sensitivity analysis can then be carried out to diminish these errors. Error sensitivity represented by the Jacobin matrix shows that $\varepsilon_{c e}$ is extremely sensitive to $\delta b_{1}$. Moreover, in order to obtain the required accuracy, standard. deviation caused by $\delta b_{1}$ is limited within $0.02 \mathrm{~mm} / \mathrm{m}$, tolerance of $\delta b_{1}$ should precisely be $0.0449 \mathrm{~mm}$. Consequently, uncontrollable errors caused by the UPS chains of the 5-dof parallel tripod head, which can not be compensated by the theoretical method, need more stringent restrictions in manufacturing and assembly stages.
\end{abstract}

\section{Introduction}

Vision system is a major way to obtain information from outside, under the background of computer technology and artificial intelligent technology, computer vision comes to be a new subject, it has began to develop towards strong practicability, such as active vision, active measurement and multisensor fusion. For the precise forecasting, tracking, detection and segmentation respecting target, the research and invention of vision tripod head have got some achievement. Parallel mechanism [1]are utilized widely in computer vision tripod head due to their advantages of few drive elements, low cost and compact structure[2]. The 5-dof visual monitoring parallel tripod head based on TriVariant mechanism is proposed in this paper, it has the advantages of simple and compact structure, strong bearing capacity, well stability, small accumulated error[3], error can be compensated and large working space of end effector. Besides, the moving platform can change and adjust the motion states by constraint driven limb structure resulted from good dexterity, driving limb fails to impose constraint and interference on moving platform. Furthermore, kinematics limb is relatively less and the drive near the base is easy to control. Nevertheless, in order to obtain clearer object in visual monitoring process, geometric precision of mechanism need higher requirements[4-5]. Therefore, the geometric precision analysis[6] is quite important in terms of 5dof parallel tripod head. This paper puts forward a error modeling[7] methodology based on screw[8] and establishes a mapping model between geometric error and end effector's pose error for 5-dof parallel mechanism by integrated modeling method to analyze the influence of geometric errors on end effector's pose error.Besides, clearly separate the geometric error source of effector's uncontrollable error[9].

\section{Architecture description and degree of freedom analysis}

Architecture description. The 5-dof parallel tripod head mentioned in section 1 is 2-UPS\&UPU structure, which is shown in Figs. 1. This mechanism consists of a base, a moving platform(end effector), a UPU chain and two UPS chains, these three limbs connecting the moving platform with the base. 


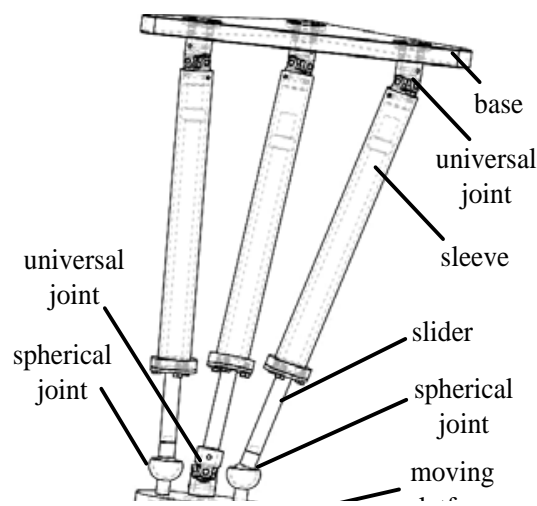

Fig.1 Sturcture of 2ups\&upu mechanical leg

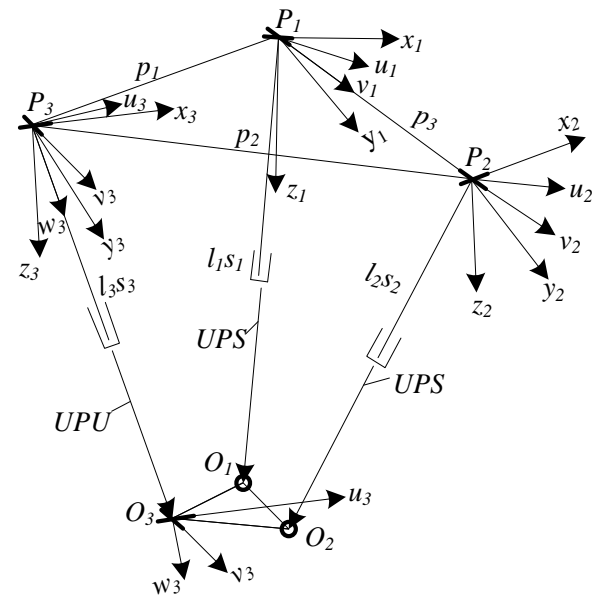

Fig. 2 Kinematic model of the 2-UPS\&UPU parallel mechanism

As presented in Fig. 2, which is rotated from axis $x_{i}$ by $\varphi_{i}$ and then rotated from axis $v_{i}$ by $\gamma_{i}$, the rotation matrix can be expressed as

$$
\begin{aligned}
& R_{i}=\operatorname{Rot}\left(x_{i}, \varphi_{i}\right) \operatorname{Rot}\left(v_{i}, \gamma_{i}\right) \\
& =\left[\begin{array}{ccc}
\cos \gamma_{i} & 0 & \sin \gamma_{i} \\
\sin \varphi_{i} \sin \gamma_{i} & \cos \varphi_{i} & -\sin \varphi_{i} \cos \gamma_{i} \\
-\cos \varphi_{i} \sin \gamma_{i} & \sin \varphi_{i} & \cos \varphi_{i} \cos \gamma_{i}
\end{array}\right] \\
& =\left[\begin{array}{lll}
u_{i} & v_{i} & w_{i}
\end{array}\right] \quad i=1,2,3
\end{aligned}
$$

Where $\boldsymbol{u}_{\boldsymbol{i}}, \boldsymbol{v}_{\boldsymbol{i}}, \boldsymbol{w}_{\boldsymbol{i}}$ is unit vector. The orientation of each limb can be denoted by

$$
\gamma_{i}=\arcsin \left(w_{i x}\right), \varphi_{i}=\arctan \left(\frac{-w_{i y}}{w_{i z}}\right)
$$

In proposed kinematic model, $\mathrm{O}_{3}$ is chosen as the reference point that can provide necessary input information to servo control and provide essential mathematical model to subsequent analysis.

The closed-loop constraint equations under the fixed coordinate frame $P_{3}-x_{3} y_{3} z_{3}$ can be obtained by

$$
\begin{gathered}
r_{3}=l_{3} s_{3} \\
r_{3}=p_{i}+l_{i} s_{i}-o_{i}=p_{i}+l_{i} s_{i}-R_{3} o_{0 i} i=1,2
\end{gathered}
$$

where $l_{i}, s_{i}$ represents length and unit vector of limb $\mathrm{i}$ respectively, and $o_{0 i}$ indicate the position vector of point $O_{i}$ under the moving coordinate frame $O_{3}-u_{3} v_{3} w_{3}$. The $l_{3}$ and $s_{3}$ can be derived based on Eqs. (3) and (4):

$$
\begin{aligned}
& l_{3}=\left\|r_{3}\right\| \\
& s_{3}=\frac{r_{3}}{l_{3}}
\end{aligned}
$$

According to Eqs. (2), orientation $\gamma_{3}$ and $\varphi_{3}$ of UPU chain can be calculate. Rotation matrix $R_{3}$ can be obtain from Eqs. (1), we can get the length of UPS chain $l_{i}$ through taking $R_{3}$ into Eqs. (4) 
then we have

$$
l_{i}=\left\|r_{i}+o_{i}-p_{i}\right\| \quad i=1,2
$$

$$
w_{i}=\frac{r_{i}+o_{i}-p_{i}}{l_{i}} \quad i=1,2
$$

Orientation $\varphi_{i}, \theta_{i}$ and rotation matrix $R_{i}(\mathrm{i}=1,2)$ for UPScan be represented Based on Eqs (2).

\section{Error sources analysis and error modeling}

For error modeling, establishing reference coordinate frame $\left\{R_{P 3}\right\}$ with origin point $P_{3}$ in the base, $z$-axis is perpendicular to plane $P$ and the direction of $x$-axis points middle point of $P_{1} P_{2}$. Moreover, instantaneous reference coordinate system $\left\{R_{O 3}\right\}$ can be obtained whose coordinate axis is parallel to that of $\left\{R_{P 3}\right\}$ instantaneously. In the ith UPS chain $(i=1,2)$, system $\left\{R_{0 . i}\right\}$ can be got through the system $\left\{R_{P 3}\right\}$ is rotated about z-axis by $\beta_{i}$. In $U P U$ chain, reference coordinate frame $\left\{R_{0.3}\right\}$ coincides with the system $\left\{R_{P 3}\right\}$. For the sake of describing the joint geometry error of each limb $i$ $(i=1,2,3)$, the origin point $P_{j a . i}$ of body-fixed coordinate frame $\left\{R_{j a . i}\right\}$ fixed on the parts consisting of the joint, $z_{j a . i}$-axis coincides with the joint axis, $x_{j a . i}$-axis is perpendicular to $z_{j a+1 . i}$-axis. Let point $P_{1 . i}$ is the intersection point of $z_{1 . i}$-axis and $x_{1 . i}$-axis, then $P_{j a . i}$ is intersection point of $z_{j a . i}$-axis and $x_{j a-1 . i}$-axis.

$$
\begin{aligned}
& \$_{t}=\sum_{j a=1}^{6} \Delta \theta_{\text {a.ja.i }} \hat{\$}_{t a . j a . i}+\$_{G . i}, \quad i=1,2
\end{aligned}
$$

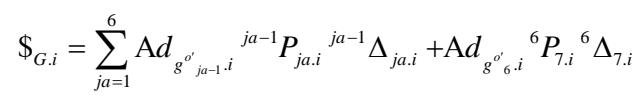

$$
\begin{aligned}
& \$_{t}=\sum_{j a=1}^{5} \Delta \theta_{a . j a .3} \hat{\$}_{t a . j a .3}+\$_{G .3} \\
& \$_{G .3}=\sum_{j a=1}^{5} \mathrm{~A} d_{g^{\sigma^{\prime}} j-1.3}{ }^{j a-1} P_{j a .3}{ }^{j a-1} \Delta_{j a .3}+\mathrm{A} d_{g^{o_{3}} .3}{ }^{5} P_{6.3}{ }^{5} \Delta_{6.3}
\end{aligned}
$$

Doing inner product simultaneously both sides of Eqs. (9) by $\$_{w a, 2, K}$ and then by $\$_{w a, 3, K}$, the general error model for UPS chain are given by

$$
\begin{aligned}
& J_{x a}=\left[\begin{array}{llll}
\$_{w a .1 .1}^{T} & \$_{w a .1 .2}^{T} & \$_{w a .3 .1}^{T}{ }^{T}{ }_{w a .3 .2 .2}^{T}
\end{array}\right]^{T}
\end{aligned}
$$

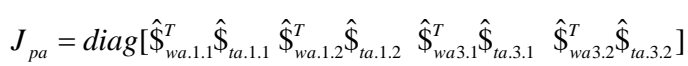

$$
\begin{aligned}
& \hat{\mathbf{S}}_{\text {wa.1.1. }}=\left[\begin{array}{c}
s_{2.1} \\
o_{1} \times s_{2.1}
\end{array}\right], \hat{\$}_{\text {wa.1.2. }}=\left[\begin{array}{c}
s_{2.2} \\
o_{2} \times s_{2.2}
\end{array}\right], \hat{\$}_{\text {wa.3.1. }}=\left[\begin{array}{c}
s_{3.1} \\
0
\end{array}\right], \quad \hat{\$}_{\text {wa.3.2. }}=\left[\begin{array}{c}
s_{3.2} \\
0
\end{array}\right] \\
& \hat{\$}_{t a .1 .1}=\left[\begin{array}{c}
\left(o_{1}-l_{3} s_{3.1}\right) \times s_{1.1} \\
s_{1.1}
\end{array}\right] \hat{\Phi}_{t a .1 .2}=\left[\begin{array}{c}
\left(o_{2}-l_{3} s_{3.2}\right) \times s_{1.2} \\
s_{1.2}
\end{array}\right], \hat{\Phi}_{t a .3 .1}=\left[\begin{array}{c}
s_{3.1} \\
0
\end{array}\right] \hat{\$}_{t a .3,2}=\left[\begin{array}{c}
s_{3.2} \\
0
\end{array}\right]
\end{aligned}
$$

The error model of UPU chain is derived through performing inner product simultaneously both sides of Eqs. (11) by $\$_{\text {wa.3.3 }}$ and then by $\$_{\text {wc.1.3. }}$

$$
\begin{aligned}
& \hat{\$}_{w a .3 .3}{ }^{T} \$_{t a}=\hat{\$}_{w a .3 .3 .3}{ }^{T} \hat{S}_{t a .3 .3} \Delta \theta_{a}+E_{a} \varepsilon_{a} \\
& \hat{\$}_{w c .1 .3}{ }^{T} \Phi_{t c}=\hat{\Phi}_{w c .1 .3}{ }^{T} \hat{\Phi}_{t c .1 .3} \Delta p_{c} \\
& \hat{\$}_{w a .3 .3}=\left[\begin{array}{c}
s_{3.3} \\
0
\end{array}\right], \hat{\$}_{t a .3 .3}=\left[\begin{array}{c}
s_{3.3} \\
0
\end{array}\right], \hat{\$}_{w c .1 .3}=\left[\begin{array}{c}
0 \\
n_{1}
\end{array}\right], \hat{\oint}_{t c .1 .3}=\left[\begin{array}{c}
-l_{3} \times n_{1} \\
n_{1}
\end{array}\right]
\end{aligned}
$$

where $n_{1}=s_{1.3} \times s_{2.3}$.

the general error model of parallel mechanism can be expressed as

$$
\begin{gathered}
J_{a} \$_{t a}=J_{p a} \Delta \theta_{a}+E_{a e} \varepsilon_{a e} \\
J_{c} \$_{t c}=E_{c e} \varepsilon_{c e}
\end{gathered}
$$

in which $\varepsilon_{a e}$ and $\varepsilon_{c e}$ is the given independent geometric error sources. Some items of Eq. (20) and (21) can be explained as follows: 


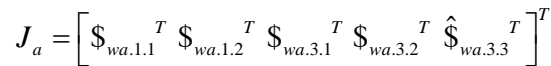

$$
\begin{aligned}
& J_{c}=\left[\hat{\$}_{w c .1 .1 .3}{ }^{T}\right]^{T}
\end{aligned}
$$

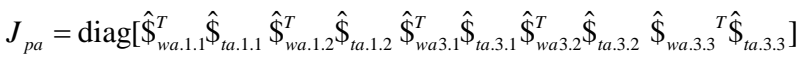

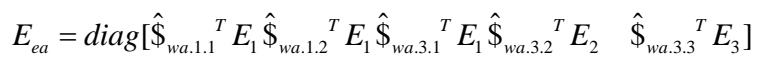

$$
\begin{aligned}
& E_{k}=\left[\mathrm{A} d_{g_{i, i}^{o}}{ }^{0} P_{1, i} \mathrm{~A} d_{g_{i, i}^{o}}{ }^{1} P_{2, i} . . \mathrm{A} d_{g_{5, i}^{o}}{ }^{5} P_{6, i}\right], k=1,2,3
\end{aligned}
$$

when $k=3$, that is to say, the analyzed object is $U P U$ chain

$$
\begin{aligned}
& E_{c e}=\operatorname{diag}\left[\hat{\$}_{w c .1 .3}{ }^{T} E_{k}\right] \\
& \varepsilon_{a e}=\left(\begin{array}{lllll}
\varepsilon_{1}{ }^{T} & \varepsilon_{2}{ }^{T} & \varepsilon_{3}{ }^{T} & \varepsilon_{4}{ }^{T} & \varepsilon_{5}{ }^{T}
\end{array}\right)^{T} \\
& \varepsilon_{c e}=\left(\begin{array}{llll}
\varepsilon_{a e}{ }^{T} & \varepsilon_{6}{ }^{T}
\end{array}\right)^{T}
\end{aligned}
$$

In light of Eqs. (19), error screw $\$_{t a}$ can be compensated by kinematic calibration that adjusting kinematic error of active joints, let $\$_{t a}=0$, we have

$$
\Delta \theta_{a}=-J_{p a}^{-1} E_{a e} \varepsilon_{a e}
$$

However, error screw $\$_{t c}$ can not be compensated. Thus, the geometric error sources $\varepsilon_{e c}$ of $\$_{t c}$ should be paid more attentions in manufacturing and assembly stage in order to reduce or eliminate the effect to pose error.

\section{Sensitivity analysis}

Sensitivity analysis model. To investigate the influences of each geometric error on the pose accuracy of the moving platform, a probability model should be proposed. $J_{c} \$_{t c}=E_{c e} \varepsilon_{c e}$ is modified into a unified form and then simultaneously multiplying both side of the equation by $J_{c}{ }^{-1}$, we can obtain

$$
\varepsilon=J_{c e} \varepsilon_{c e}
$$

Error vector of the end is denoted by $\varepsilon$, geometric error sources vector is denoted by $\varepsilon_{c e}$, and error transformation matrix between the two vector is denoted by $J_{c e}$.

Now some sensitivity indices can evaluate the influences of each geometric error on the pose errors that can not be uncompensated of moving platform, which are

$$
\begin{aligned}
& \bar{\mu}_{k}=\left(\int_{V} \mu_{k} d V\right) / V \\
& \mu_{k}=\sqrt{\sum_{i=1}^{3} J_{c e i j}{ }^{2}}
\end{aligned}
$$

note that $\mu_{k}$ denotes the sensitivity of $\varepsilon$ relative to $\varepsilon_{c e}$, which represent the standard deviation of $\varepsilon$ caused by the unit standard deviation of $\varepsilon_{c e} \cdot \bar{\mu}_{k}$ denotes the universe value of $\mu_{k}$.

Sensitivity analysis. Based on the indices defined by Eqs.(31), the sensitivity analysis can be carried out for the mechanism shown in Fig.2. The investigated workspace, which takes $\mathrm{O}_{3}$ as reference point, is a cylinder area of $\phi 500 \mathrm{~mm} \times 220 \mathrm{~mm}$, the dimension parameters of base and moving platform are identified as

$$
o_{i}=\left[\begin{array}{lll}
85(-1)^{i} & 45 & 0
\end{array}\right]^{T}, p_{i}=\left[\begin{array}{lll}
425(-1)^{i} & 240 & 0
\end{array}\right]^{T}
$$

the error sources of uncontrollable orientation error on end effector can be simplified as

$$
\varepsilon_{c e}=\left[\begin{array}{lll}
\delta b_{1} & \delta b_{2} & \delta a
\end{array}\right]^{T}
$$

The maximum value is $0.0352 \mathrm{~mm} / \mathrm{m}$, minimum value is $0.0329 \mathrm{~mm} / \mathrm{m}$, and average value reaches $0.0340 \mathrm{~mm} / \mathrm{m}$. The global sensitivity coefficient that $\varepsilon_{c e}$ is about geometrical parameter errors $\delta b_{1}, \delta b_{2}$ and $\delta a$ are $\bar{\mu}_{k b 1}=1.334 \mathrm{rad} / \mathrm{m}, \bar{\mu}_{k b 2}=1.183 \mathrm{rad} / \mathrm{m}, \bar{\mu}_{k a}=1.291 \mathrm{rad} / \mathrm{m}$, As shown in Fig. 4, $\delta b_{1}$ has the greatest influence on $\varepsilon_{c e}$. based on $3 \sigma$ criterion, the tolerance of $\delta b_{1}$ should be calculated as $T\left(\delta b_{1}\right)= \pm(3 \times 0.02 / 1.334)=0.0449 \mathrm{~mm}$. 
Table 1 The effects of constraint limb on the position error of end effector

\begin{tabular}{|c|c|c|}
\hline Geometric error & $\begin{array}{c}\text { Standard } \\
\text { deviation } \\
/ \mathrm{mm}\end{array}$ & $\begin{array}{c}\text { Maximum orientation } \\
\text { error of the end effector } \\
\mathrm{mm} / \mathrm{m}\end{array}$ \\
\hline$\delta b_{1}$ & 0.01 & 0.0352 \\
\hline$\delta b_{2}$ & 0.01 & 0.0329 \\
\hline$\delta a$ & 0.02 & 0.0340 \\
\hline
\end{tabular}

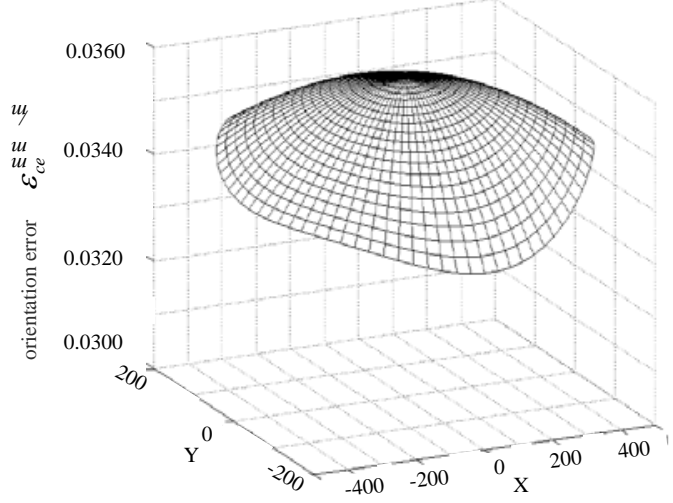

Fig. 4 Distribution of uncontrolled error

\section{Conclusions}

i) Comparing with the existing error model based on space vector chain and vector differential method, the integrated error model, which is on account of variational screw for parallel mechanism, reflects more accurately nonlinear mapping relation between pose error and geometric error.

ii) The sensitivity indice $\mu_{k}$ can be defined in the sense of statistics. The error brought by the two non-intersecting axes of universal joint, the position error result from the zero point of universal joint and the error in torsional angle of sleeve guide, all of which have great influences on the end effector's errors by the sensitivity analysis.

iii) According sensitivity analysis, the error sources $\delta b_{1}, \delta b_{2} \delta a \quad \delta p$ and $\delta o$ have remarkable effect on the uncontrollable error, and $\varepsilon_{c e}$ is sensitive to the linear error result from $\delta b_{1}$. Thus, the tolerance of $\delta b_{1}$ must meet the design requirements in advance.

\section{Acknowledgements}

The research was supported by The Youth Natural Science Foundation of Hebei, China (Grant: F2012203031) and The Excellent Going Abroad Experts' Training Program in Hebei Province, China.

\section{References}

[1] YAO Rui,TANG Xiaoqiang,LI Tiemin. Error Analysis and Distribution of 6-SPS and 6-PSS Reconfigurable Parallel Manipulators. Tsinghua Science and Technolocy. vol. 15, no.5, pp. 547-554, 2010.

[2] Wang Gengxiang, Yuan Daning, Liu Hongzhao. Kinematics Analysis of Spatial 4-SPS/CU Parallel Mechanism. Transactions of the Chinese Society for Agricultural Machinery. vol. 43, no. 3, pp. 208-212,2012.

[3] Chen Xiulong, Sun Xianyang,Deng Yu. Kinematics Analysis of 4-UPS_RPS Spatial 5-DOF Parallel Mechanism. Transactions of the Chinese Society for Agricultural Machinery. vol. 44, no.8, pp. 257-261, 2013.

[4] MEI F, ZHAO XH. Accuracy analysis of 3-DOF parallel manipulator. Journal of Tianjin 
University of Technology. vol. 26, no. 4, pp.15-17,2010.

[5] Tan Xingqiang, Zhang Jian. Motion error modeling and compensating for 6_PUS parallel robot of wind tunnel support system. Transactions of the Chinese Society for Agricultural Machinery. vol.45, no.4, pp.334-346, 2014.

[6] LI Ruiqin, YANG Bin. Error modeling of 3-RSR parallel robot based on D-H transformation matrix. Journal of Measurement Science and Instrumentation. vol.5,no.3, pp.53-60,2014.

[7] Li Yongquan, Zhang Lijie,Guo Zhimin. Error Modeling and Sensitivity Analysis of Spherical 5R Parallel Manipulator Based on D-H Matrix.China Mechanical Engineering. vol. 23, no. 12, pp. 1470-1474, 2012.

[8] HUANG Peng, WANG jingsong, WANG liping. Identification of structure errors of 3-PRS-XY mechanism with Regularization method.Mechanism and Machine Theory. vol. 46, no. 7, pp. 927-944, 2011.

[9] Wu Mengli, Wang Liwen. Statics Optimum Design of a New Parallel Robot.China Mechanical Engineering. vol. 21, no. 19, pp. 2308-2313, 2010. 